\title{
INTEGRAÇÃO ESPACIAL DOS MERCADOS EXPORTADORES DE MEL NATURAL NO BRASIL
}

\author{
Manoel Pedro da Costa Júnior* \\ pedrojunior_noronha@yahoo.com.br
}

\author{
Ahmad Saeed Khan** \\ saeed@ufc.br
}

Eliane Pinheiro de Sousa***

pinheiroeliane@hotmail.com

\section{Patrícia Verônica Pinheiro Sales Lima** pvpslima@gmail.com}

* Instituto Federal de Educação, Ciência e Tecnologia do Sertão Pernambucano - PE / Brasil ** Universidade Federal do Ceará - CE / Brasil

*** Universidade Regional do Cariri - CE / Brasil

http://dx.doi.org/10.1590/1413-2311.023.55682

Recebido em 15/05/2015

Aprovado em 15/02/2017

Disponibilizado em 31/05/2017

Avaliado pelo sistema "double blind review"

Revista Eletrônica de Administração

Editora-chefe: Andrea Oltramari

ISSN: 1413-2311 (versão "on line")

Editada pela Escola de Administração da Universidade Federal do Rio Grande do Sul Periodicidade: Quadrimestral

Sistema Requerido: Adobe Acrobat Reader

\section{RESUMO}

A análise de integração de mercados reveste-se de importância para o desenvolvimento econômico de um país. Em face dessa relevância e do papel desempenhado pelo mel natural na economia brasileira, torna-se fundamental compreender como ocorre a integração desse produto nos principais estados brasileiros exportadores. Neste sentido, o estudo buscou investigar o processo de integração espacial entre os principais mercados brasileiros exportadores de mel natural: Rio Grande do Sul, Santa Catarina, Piauí, Ceará e São Paulo. Utilizaram-se dados mensais obtidos por meio da base de dados do Ministério de 
Desenvolvimento, Indústria e Comércio Exterior (MDIC) para o período de janeiro de 2002 a julho de 2011. Para investigar as relações entre esses mercados, fez-se uso dos métodos empregados em séries temporais, como os testes de raiz unitária e de cointegração de Johansen, análise do modelo vetorial de correção de erro e teste de exogeneidade. Os resultados indicaram a presença de cointegração entre os mercados brasileiros exportadores de mel natural. No entanto, com base no teste sobre o coeficiente de relacionamento de longo prazo, verificou-se que a Lei do Preço Único não foi totalmente confirmada, já que se rejeitou a hipótese nula de perfeita integração espacial entre esses mercados. Ademais, o teste de exogeneidade fraca indica que o mercado de mel natural do Rio Grande do Sul atua como mercado central na formação de preços.

Palavras-Chave: Integração de mercados; mel natural. Lei do Preço Único. Séries de tempo.

\title{
SPATIAL INTEGRATION OF RAW HONEY EXPORT MARKETS IN BRAZIL
}

\begin{abstract}
Market integration analysis is important for the economic development of a country. In the context of this relevance and of the role played by raw honey in the Brazilian economy, it's fundamental to understand how the integration of this product takes place in the main Brazilian export States. In this sense, the study aimed at investigating the spatial integration process among the main Brazilian raw honey export markets: Rio Grande do Sul, Santa Catarina, Piauí, Ceará and São Paulo. Monthly data obtained by means of the database of the Ministry of Development, Industry and Foreign Commerce (MDIC) for the period from January 2002 to July 2011 was used. In order to investigate the relationships among these markets, the methods employed in time series, such as unit root and Johansen cointegration tests, analysis of the vector model of error correction and exogeneity test were used. The results indicated the presence of cointegration among the Brazilian raw honey export markets. However, based on the test about the long-run relationship coefficient, it was observed that the Law of One Price was not completely confirmed, as the null hypothesis of perfect spatial integration among these markets was rejected. In addition, the testing of weak exogeneity indicates that the Rio Grande do Sul's raw honey market acts like a central market in the formation of prices.
\end{abstract}


Keywords: Market integration. raw honey. Law of One Price. Time series.

\section{INTEGRACIÓN ESPACIAL DE LOS MERCADOS EXPORTADORES DE MIEL NATURAL EN BRASIL}

\section{RESUMEN}

El análisis de integración de mercados se viste de importancia para el desarrollo económico de un país. En vista de esa relevancia y del rol desempeñado por la miel natural en la economía brasileña, se hace fundamental comprender cómo ocurre la integración de ese producto en los principales estados brasileños exportadores. En este sentido, el estudio buscó investigar el proceso de integración espacial entre los principales mercados brasileños exportadores de miel natural: Rio Grande do Sul, Santa Catarina, Piauí, Ceará y São Paulo. Se utilizaron datos mensuales obtenidos por medio de la base de datos del Ministerio de Desarrollo, Industria y Comercio Exterior (MDIC) para el periodo de enero de 2002 a julio de 2011. Para investigar las relaciones entre esos mercados, se hizo uso de métodos empleados en series temporales, tales como los testes de raíz unitaria y de cointegración de Johansen, análisis del modelo vectorial de corrección de error y test de exogeneidad. Los resultados indicaron la presencia de cointegración entre los mercados brasileños exportadores de miel natural. Sin embargo, con base en el test sobre el coeficiente de relacionamiento de largo plazo, se verificó que la Ley del Precio Único no ha sido totalmente confirmada ya que se rechazó la hipótesis nula de perfecta integración espacial entre esos mercados. Además, el test de exogeneidad débil indica que el mercado de miel natural de Rio Grande do Sul actúa como mercado central en la formación de precios.

Palabras clave: Integración de mercados. miel natural. Ley del Precio Único. Series de tiempo.

\section{INTRODUÇÃO}

A produção apícola mostra-se bastante eficiente, visto que além de gerar resultados econômicos satisfatórios, consegue contribuir para a preservação do meio ambiente, em decorrência da necessidade de existir florada suficiente para o processo de produção do mel, 
bem como os demais produtos gerados (pólen, geleia real etc.). Segundo Freitas, Khan e Silva (2004), a atividade apícola atua de forma sustentável sobre o campo social, econômico e ambiental. É importante ressaltar que um dos componentes que colaboram para níveis de rentabilidade mais elevados, é o baixo nível de investimentos em equipamentos e tecnologia, ratificando a necessidade de flora abundante, onde o apiário é instalado.

O Brasil possui fatores que permitem realizar previsões otimistas para alcançar níveis de produção e comercialização capazes de se tornar líder entre as nações produtoras de mel. Tal possibilidade pode ser fruto da larga extensão territorial e diversidade de floras. Para Matos (2005, p.24):

O Brasil tem grande potencial para se tornar o maior produtor e exportador de mel natural: o mel brasileiro é relativamente pouco conhecido no mercado externo e o País dispõe de grande diversidade de flora, extenso pasto apícola, condições climáticas apropriadas e abelhas africanizadas, que são mais resistentes a pragas e doenças, sendo raro o uso de qualquer medicamento para o tratamento das abelhas.

Ressalta-se que o mel brasileiro conquistou maiores níveis de aceitação no mercado internacional devido à adequação a processos capazes de minimizar efeitos que gerem redução na qualidade do produto, com destaque para presença de defensivos agrícolas. Quanto ao cumprimento das especificidades da demanda dos consumidores que exigem alimentos que não possuam vestígios de resíduos redutores de qualidade, o Brasil, se comparado com a China (umas das nações que mais produzem e comercializam mel), possui vantagens, que podem ser atribuídas principalmente às floras (MATOS, 2005).

Segundo dados da Organização das Nações Unidas para a Agricultura e Alimentação (FAO, 2011), nas últimas décadas do século XX e na primeira deste século, o Brasil esteve presente entre os 11 países que mais exportaram mel natural. Reportando-se para especificação de nuanças a respeito do panorama interno brasileiro, conforme as informações do Ministério de Desenvolvimento Indústria e Comércio / Secretaria de Comércio Exterior (MDIC/SECEX, 2011), desenhado durante o período do ano de 2000 a 2010, foi apontada com 36,12\%, a região Sudeste como detentora da maior parcela do total exportado de mel natural - em termos absolutos, o volume superior a 57,9 mil toneladas, capaz de gerar uma receita de 126 milhões de dólares. A região Sul alcançou o segundo lugar com o envio de 52,2 mil toneladas, gerando 111,4 milhões de dólares, representando um percentual de 32,55\% do total comercializado internacionalmente de mel natural pelo Brasil. O Nordeste, por sua vez, com 
31,27\%, aparece em terceiro lugar com 50,2 mil toneladas, gerando 109,4 milhões de dólares. Com totais bem inferiores aos comparados com as regiões supracitadas, o Centro-Oeste e o Norte brasileiros, apresentaram, respectivamente, 97 e 9,5 toneladas exportadas, com receita total de 266,4 e 10 mil dólares, sinalizando inexpressiva participação no total de mel natural exportado pelo Brasil, já que os referidos volumes de exportação alcançam pífio $0,01 \%$.

Categorizados como conjunto de estados com maior volume de mel natural exportado, considerando o período referente à primeira década do século XXI e segundo a base de dados do MDIC/SECEX (2011), Rio Grande do Sul, Piauí, Ceará, Santa Catarina e São Paulo alcançaram inserção nesta pesquisa.

Dada a relevância desempenhada pelo mel natural na economia brasileira, torna-se importante compreender como acontece o processo de integração desse produto nos principais estados exportadores. De acordo com Meyer (2004), a integração de mercado pode ser definida como o grau de transmissão de preços entre mercados espacialmente separados. As commodities têm sido alvos frequentes dos estudos de integração de mercados, que mensura se os choques de oferta e demanda em uma dada região podem ser transmitidos para outra. Mól e Aldatz (2014) destacam que a integração de mercados gera relevantes implicações sobre os preços e operações comerciais e que o comportamento dos preços atua como um importante indicador de performance do mercado.

Estudos dessa natureza que avaliam o processo de transmissão de preços têm sido realizados para várias culturas agropecuárias no Brasil, como, por exemplo, algodão, cacau, camarão, carnes, castanha de caju, soja, etanol, frutas, fumo, trigo etc. Entretanto, não se encontrou na literatura essa aplicação para os mercados brasileiros exportadores de mel natural, apesar da expressiva relevância desempenhada por esse produto para a economia brasileira.

Portanto, a análise das variações de preços de exportação do mercado brasileiro de mel natural permitirá reunir uma série de informações que possibilitará compreender com maior clareza sobre como tais preços são constituídos, se a Lei do Preço Único (LPU) pode ser aplicada entre esses mercados e identificar o mercado que é formador de preços, ou seja, o estado que atua como mercado central, servindo como referência para os demais estados exportadores. Salienta-se ainda que este estudo fornecerá subsídios que ajudarão no processo de formulação de políticas de comercialização, com o intuito de conquistar maior competitividade do mel natural produzido no Brasil. 


\section{REFERENCIAL TEÓRICO}

De acordo com Faminow e Benson (1990), integração de mercados diz respeito ao processo pelo qual acontece a interdependência de preços. Usando os termos definidos por Nogueira et al. (2005), a integração espacial de mercados corresponde ao grau de comovimentação dos preços em diferentes localidades. Essa análise pode ser feita tanto em diversas regiões de um mesmo país quanto em países distintos. Tal definição provém da Lei do Preço Único (LPU), embasada sob a hipótese de ausência de custos de transação, bens homogêneos, ausência de barreiras tarifárias e não tarifárias e informação perfeita. Essa lei fundamenta-se no princípio da arbitragem de mercadorias (KRUGMAN; OBSTFELD, 2005).

Conforme Ardeni (1989), se houver arbitragem, o preço de um produto tenderá a aumentar no mercado com menor preço, decorrente do acréscimo da quantidade demandada, enquanto o preço tenderá a diminuir no mercado com maior preço, devido ao excesso da quantidade ofertada. Esse processo de arbitragem permanecerá até que os preços se igualem nos dois mercados.

Em outras palavras, o conceito de arbitragem se reporta à disposição de comprar um bem em um local qualquer, no tempo ou na forma com a transação oferecendo menor dispêndio financeiro, e, em seguida, comercializá-lo no mercado que pode gerar maior receita, considerando os custos de transferência. Desta forma, as ações dos arbitradores espaciais assegurarão que os preços de um bem homogêneo, em quaisquer duas localidades, diferirão, no máximo, ao custo de se mudar o bem ou mercadoria de uma região com o menor preço para outra região com o maior preço.

Portanto, o preço de um bem qualquer não pode diferir entre os países, quando for expresso na mesma moeda. Em um dado instante, caso o preço de certo produto no país “ $A$ ” exceda ao preço em moeda internacional (por exemplo, em dólar) no país "B", haverá uma oportunidade de ganho para os agentes econômicos através da compra do bem no primeiro país para revenda no segundo país. Em termos algébricos, pode ser representado por: $P_{i t}=E_{t} P_{i t}^{*}$, em que: $P_{i t}$ : preço do i-ésimo bem no país $\mathrm{A} ; P_{i t}^{*}$ : preço do i-ésimo bem no país B (mercado internacional); e $E_{t}$ : taxa de câmbio nominal.

Para Fackler e Goodwin (2001), a LPU pode ser manifestada nas seguintes formas:

(i) LPU Fraca: ocorre diante da condição de arbitragem espacial;

(ii) LPU Agregada: enunciada em termos de índice de preços e conhecida como Paridade do Poder de Compra (PPC); e 
(iii) LPU Forte: considera que o comércio seja contínuo e assegura a condição de arbitragem. Essa última forma é a mais comumente verificada.

\section{REVISÃO DE LITERATURA}

Com base na literatura, verifica-se que existem mais estudos que procuraram avaliar a interdependência dos preços de produtos agrícolas entre mercados internos e externos do que entre mercados regionais de um mesmo país. Entretanto, os pressupostos da Lei do Preço Único também são válidos entre diferentes regiões de um mesmo país, conforme se observa, por exemplo, nos trabalhos de Nogueira et al. (2005), para mercados brasileiros de café arábica (Minas Gerais e São Paulo), no período de 1996 a 2000; e de Chiodi (2006), para mercados de milho (entre os estados do Centro-Oeste e entre os estados sulistas de Santa Catarina e do Rio Grande do Sul), no período de 1996 a 2004.

Em contrapartida, apesar de se verificar o relacionamento entre preços de commodities, seja entre mercados domésticos e internacionais ou entre regiões de um mercado interno, os pressupostos da LPU podem não ser perfeitamente verificados. Nesse contexto, ressalta-se, por exemplo, o estudo desenvolvido por Rosado (2006) em torno do mercado brasileiro de suínos no período de janeiro de 1980 a março de 2005. Os resultados sinalizaram que os preços de suínos nos estados do Mato Grosso, Mato Grosso do Sul, Goiás, Rio de Janeiro, Minas Gerais, Paraná, Rio Grande do Sul, Santa Catarina e São Paulo compartilharam uma única tendência de comportamento ao longo do tempo. Entretanto, constatou-se que o padrão de relacionamento estabelecido entre os nove estados integrados denota que não houve localidade dominante do comportamento no mercado, bem como inexistência de integração perfeita entre todos os estados.

O estudo realizado por Sousa e Campos (2008) buscou testar a validade da Lei do Preço Único na relação entre os preços internos do algodão em pluma, representado pelo posto de São Paulo, e externo, representado pela bolsa de Nova Iorque, no período de julho de 1996 a janeiro de 2008. Os resultados legitimaram que movimentos de preços no mercado brasileiro e no mercado norte-americano são significativamente relevantes no estabelecimento do padrão de equilíbrio de longo prazo. Entretanto, a hipótese de perfeita integração entre esses mercados foi rejeitada, não se configurando na transmissão completa de alterações de preços de um mercado para outro, revelando, portanto, que a LPU não foi perfeitamente verificada no mercado de algodão nesse período analisado. 
A validade da Lei do Preço Único também foi testada nos mercados brasileiros de soja por Sousa e Campos (2009). Os resultados demonstraram que as elasticidades de transmissão de preços entre Paraná e Rio Grande do Sul e entre Mato Grosso e Rio Grande do Sul indicaram que variações em um mercado, em longo prazo, foram repassadas quase que totalmente para o outro mercado avaliado, durante o período de janeiro de 2001 a fevereiro de 2008, ressaltando assim, a predominância da Lei do Preço Único nesses mercados. No entanto, tais mercados não podem ser considerados perfeitamente integrados, pois a hipótese de perfeita integração entre eles foi rejeitada. Assim, a Lei do Preço Único não foi perfeitamente constatada para o mercado de soja nesses estados analisados durante esse período enfocado.

Coronel et al. (2010) analisaram a integração e transmissão de preços do trigo entre os mercados argentino e internacional no período de janeiro de 1994 a abril de 2009. Os principais resultados elencados pelos autores foram que as velocidades de resposta das variáveis a uma dada situação de desequilíbrio de curto prazo no processo de ajuste de longo prazo não foram estatisticamente iguais para o mercado argentino e o mercado internacional de trigo no período considerado; e que os movimentos de preços no mercado argentino e no mercado internacional foram significativamente relevantes no estabelecimento do padrão de equilíbrio de longo prazo. Entretanto, apesar desses mercados serem considerados como integrados, verificou-se que a hipótese de perfeita integração entre tais mercados foi rejeitada, indicando que alteração de preços em um mercado não foi completamente transmitida ao outro no longo prazo. Com isso, a LPU não foi perfeitamente verificada no mercado do trigo nesse período analisado.

Com o intuito de verificar se os mercados de fumo internacional e nacional são integrados e determinar a magnitude em que ocorreu a transmissão de preços entre os principais estados produtores brasileiros (Bahia, Paraná, Rio Grande do Sul, Santa Catarina e Sergipe), Freitas et al. (2012) procederam a análise considerando o período de janeiro de 1999 a dezembro de 2009. No tocante ao mercado nacional, os resultados indicaram que as séries possuem relação de equilíbrio de longo prazo e que os preços recebidos pelo produtor de fumo no Paraná são mais sensíveis às variações nos preços do Rio Grande do Sul e de Santa Catarina. Quanto ao mercado internacional, os resultados revelaram que o preço do tabaco no mercado nacional apresenta alta sensibilidade às variações no preço do produto no mercado externo.

Amorim et al. (2013) buscaram identificar a integração espacial para o preço da cesta básica entre as capitais da Região Sul do Brasil para o período de janeiro de 1995 a dezembro de 
2010. Os resultados apontaram que, embora as informações tenham sido transmitidas rapidamente entre os agentes das capitais sulistas, a presença da Lei do Preço Único não é corroborada nestes mercados, ou seja, a transmissão dos preços da cesta básica não se dá completamente de uma cidade para outra.

Mól e Aldatz (2014) investigaram as relações de longo prazo do preço do camarão pago ao produtor em quatro microrregiões do estado do Rio Grande do Norte (Natal-Macaíba, Litoral Nordeste, Vale do Açu e Litoral Sul), para o período de janeiro de 2008 a dezembro de 2011. Os resultados evidenciaram que essas regiões participam das relações de cointegração e não apresentam exonegeneidade fraca, explicitando a relevância de cada microrregião na formação do preço interno. Ademais, tais mercados podem ser caracterizados como perfeitamente integrados.

Moraes (2014), por sua vez, analisou as relações de longo prazo do preço do etanol do segmento produtor nos estados brasileiros do Paraná, São Paulo, Minas Gerais, Goiás, Mato Grosso, Mato Grosso do Sul, Alagoas e Pernambuco, considerando o período de abril de 2008 a março de 2013. Os resultados apontaram que os estados de São Paulo, Paraná, Minas Gerais e Goiás são perfeitamente integrados entre si, assim como os estados da região Nordeste, representados por Alagoas e Pernambuco, também são perfeitamente integrados. Verificou-se ainda que, embora o relacionamento entre os estados analisados não seja perfeito, todos demonstraram relações com os estados nordestinos, e também com o estado do Mato Grosso.

A literatura que versa sobre análises de integração espacial de mercados de commodities, conforme destacada, é bastante vasta. No entanto, especificamente, não foram identificadas pesquisas que contemplassem o processo de integração espacial dos mercados brasileiros de mel natural. Portanto, este estudo pretende preencher essa lacuna.

\section{METODOLOGIA}

\subsection{Natureza dos dados}

Os dados utilizados neste trabalho contemplaram as séries mensais de preços de exportação (US\$/ton.) do mel natural nos estados brasileiros de São Paulo, Ceará, Piauí, Santa Catarina e Rio Grande do Sul, obtidos junto ao Ministério do Desenvolvimento, Indústria e Comércio Exterior e Secretária de Comércio Exterior (MDIC/SECEX, 2011) para o período de janeiro de 2002 a julho de 2011. Essas séries, respectivamente são representadas por: LPSP 
(Logaritmo natural do preço no estado de São Paulo), LPCE (Logaritmo natural do preço no estado do Ceará), LPPI (Logaritmo natural do preço no estado do Piauí), LPSC (Logaritmo natural do preço no estado de Santa Catarina) e LPRS (Logaritmo natural do preço no estado do Rio Grande do Sul).

\subsection{Métodos analíticos}

Para testar a presença ou não de raiz unitária na série e a ordem de integração, foi usado o teste de Dickey-Fuller Aumentado (ADF), desenvolvido por Dickey e Fuller (1981), Teste Phillips-Perron (PP) e Kwiatkowski, Phillips, Schmidt e Shin (KPSS). No teste ADF, a hipótese nula corresponde à existência de uma raiz unitária e a hipótese alternativa indica que não há raiz unitária. Segundo Margarido e Medeiros Júnior (2006), uma das vantagens em optar pelo teste ADF é o fato de poder introduzir o número de defasagens adequadas, utilizando, por exemplo, os critérios de Akaike (AIC), Schwarz (SIC) e Hannan-Quinn (HQ). No sentido de validar os resultados do teste ADF, outros dois métodos foram utilizados para se testar a presença de raiz unitária. O teste PP associa-se à pressuposição de que o resíduo possui o comportamento de um ruído branco, em que as equações estimadas para o cálculo da estatística $t$ são idênticas às equações do teste DF. O teste KPSS, apresentado por Kwiatkowski et al. (1992) apud Mayorga (2006) surge como alternativa mais eficiente relativamente à limitação de potência dos testes convencionais, em que este último tende a não rejeitar a hipótese nula com demasiada frequência. A metodologia desenvolvida por esses autores sugere uma inversão das hipóteses consideradas, assumindo assim a característica de série estacionária na hipótese nula, e, por sua vez, a hipótese alternativa descreve uma série integrada de ordem um.

Salienta-se que os testes convencionais de raiz unitária não levam em consideração o componente sazonal das séries temporais. Desta forma, para a identificação do componente sazonal, faz-se uso do teste de raiz unitária sazonal, sendo que o mais comumente utilizado é o teste HEGY, proposto em Hylleberg et al. (1990), adotado neste estudo.

Segundo Cunha (2008), quando o termo $\pi_{1}$, que representa um dos parâmetros da regressão a ser estimada para identificação da raiz unitária, for estatisticamente igual a zero, significa que a série possuirá uma raiz unitária regular e quando houver qualquer um dos outros $\pi \mathrm{i}$ 's $=0$ $(\mathrm{i}=2, \ldots, 12)$, sinalizará presença de raízes unitárias sazonais. Ademais, adota-se o teste t convencional para analisar a significância estatística de $\pi_{1}$ e $\pi_{2}$, enquanto utiliza-se o teste $\mathrm{F}$ 
para verificar a significância dos pares de raízes complexas, sendo que os valores críticos foram obtidos por Franses e Hobijn (1997).

O modelo VAR objetiva analisar as relações lineares entre cada variável e seus valores defasados, considerando os problemas básicos, porém, primordiais para realizar uma análise eficiente, que dizem respeito à conjugação de variáveis que possam melhor explicar o problema de pesquisa, bem como o total de defasagens relacionadas às variáveis do modelo. Considerando uma abordagem genérica, estruturalmente, o modelo pode ser representado desta forma: $B X_{t}=B_{0}+\sum_{i=1}^{p} B_{i} X_{t-i}+\varepsilon_{t}$, em que: $X_{t}$ : é um vetor representado por uma matriz ( $\mathrm{n} \times 1) ; \beta_{0}$ : é um parâmetro que pode assumir os seguintes valores: $\mathrm{i}=0,1,2,3, \ldots, \mathrm{p}$, matricialmente representado por: $(\mathrm{n} \times \mathrm{n})$; e $\varepsilon_{t}$ representa os termos de erros com vetor $(\mathrm{n} \times 1)$. A condição necessária para que os estimadores obtidos possuam propriedades desejáveis é que as variáveis do VAR sejam estacionárias. Caso contrário, a existência de raízes unitárias deve ser levada em consideração. Uma situação em que se pode trabalhar com séries em níveis, evitando regressões espúrias ocorre quando as séries são cointegradas.

Os Vetores de Correção de Erro (VEC) permitem a identificação das relações entre as variáveis consideradas no modelo, tanto no curto como no longo prazo, ao contrário das metodologias convencionais que analisam exclusivamente as relações no curto prazo (HAUER, 2007). Esse modelo pode ser descrito como: $\Delta Y_{t}=\prod_{1} \Delta Y_{t-1}+\prod_{2} \Delta Y_{t-2}+\cdots+\prod_{p-1} \Delta Y_{t-p+1}+\varepsilon_{t}$ A matriz $\prod\left(\begin{array}{lll}n & x & n\end{array}\right)$ pode ser definida da seguinte forma: $\Pi=\alpha \beta^{\prime}$, em que $\alpha$ : representa a matriz com indicadores da velocidade de ajustamento dos parâmetros de curto prazo; e $\beta$ : Matriz de coeficiente de cointegração de longo prazo entre as variáveis.

Os parâmetros $\alpha$ e $\beta$ são matrizes de ordem $n \times r$, em que $n$ representa o número de variáveis integrantes do modelo e $r$, o número de vetores de cointegração da matriz $\prod$. Salienta-se que o comportamento da variável $Y_{t}$ dependerá dos autovalores da matriz de longo prazo $\prod$. Para definir o número de vetores de cointegração, precisa-se do posto da matriz $\prod$. Conforme Patterson (2000, p. 620); Valls (2004, p. 34) apud Mayorga (2006, p. 61-62), tem-se os seguintes casos:

a) Se todos os autovalores de $\prod$ forem diferentes de zero $(r=n)$, esta matriz terá posto completo e, consequentemente, todos os componentes de $Y_{t}$ serão estacionários, devendo-se empregar o VAP (p) em nível; 
b) Caso todos os autovalores de $\Pi$ forem iguais a zero $(r=0)$, esta matriz será nula e, consequentemente, os componentes de $Y_{t}$ serão no mínimo I(1), devendo-se, assim, utilizar o $\operatorname{VAR}(p-1)$ em primeira diferença; e

c) Caso possuam posto reduzido, isto é, $0<r<n$, terão neste caso, $n-r$ autovalores diferentes de zero e os componentes de $Y_{t}$ serão no mínimo I(1), devendo-se adotar o VEC e nela estarão contidas $r$ relações de cointegração.

Neste estudo, foi adotado o teste de cointegração de Johansen, que estima os vetores de cointegração por meio do método de máxima verossimilhança e permite verificar a presença de vários vetores e não apenas um único vetor de cointegração. Para identificar a presença de vetores de cointegração, utilizaram-se os testes do traço e do máximo autovalor. Segundo Enders (1995, p. 391), “o teste do traço busca verificar a hipótese nula de que o número de vetores de cointegração distintos seja menor ou igual a r contra a hipótese alternativa de que o número desses vetores seja maior do que r". O teste do máximo autovalor objetiva testar a existência de exatamente $r$ vetores de cointegração contra a alternativa de existência de $r+1$ vetores.

Ademais, também foram realizados testes relacionados aos parâmetros $\beta$ e $\alpha$ com o intuito de avaliar, respectivamente, a velocidade de ajustamento referente a cada variável e observar a relevância das variáveis inseridas no universo de cointegração, por meio de testes de significância. Em outros termos, esses testes buscam, respectivamente, identificar os mercados considerados nesta análise que efetivamente compõem o equilíbrio no longo prazo, e verificar a existência de integração perfeita, confirmando a validade da hipótese da Lei do Preço Único.

De acordo com Johansen e Juselius (1990), as hipóteses a respeito dos parâmetros $\beta$ apresentam o seguinte formato: $-2 \log Q\left(H_{\mathrm{o}}\right)=-T \sum_{i=1}^{r} \log \left[\frac{\left(1-\lambda_{i}^{*}\right.}{\left(1-\lambda_{i}\right.}\right]$, para $\mathrm{i}=1, \ldots, \mathrm{r}$, em que: $\boldsymbol{T}$ : total de observações; $r$ : total de vetores de cointegração; $\lambda_{i}^{*}$ : autovalores do modelo restrito: e $\lambda_{i}$ : autovalores do modelo irrestrito.

Desta forma, caso o valor alcançado pelo parâmetro configure menor escala quando comparado com o valor crítico, não será rejeitada a validade do modelo com restrição. Em panorama distinto, ocorrendo assim, a valoração do parâmetro em maior grau que o valor crítico, rejeita-se a validade da restrição. 
A significância do teste de hipótese sobre o parâmetro $\alpha$ indica que a variável preço do mel natural no mercado analisado não pode ser considerada exógena fraca em relação ao parâmetro de longo prazo. A existência de exogeneidade fraca indica que a variável não reagirá ao desequilíbrio de equilíbrio de longo prazo. A estatística de teste de razão de verossimilhança do parâmetro $\alpha$ segue a mesma formulação do parâmetro $\beta$.

\section{RESULTADOS E DISCUSSÃO}

\subsection{Testes de raiz unitária}

Para verificar a estacionariedade das séries consideradas, empregaram-se os testes de raiz unitária de Dickey-Fuller Aumentado (ADF), de Phillips-Perron (PP) e do KPSS. Com base na Tabela 1, as séries analisadas não são estacionárias em nível, mas passam a ser estacionárias em primeira diferença, indicando que apenas uma diferenciação é suficiente para torná-las estacionárias, considerando os testes de ADF e PP com defasagens baseadas no critério de SIC (Schwarz Info Criterion) para os modelos com a presença somente de intercepto (I); intercepto e tendência (II); e ausência destes dois componentes (III).

Tabela 1 - Resultados dos testes ADF e PP em nível e em primeira diferença para as séries mensais de preços logaritmizadas do mel natural brasileiro exportado pelos estados considerados na análise, janeiro de 2002 a julho de 2011.

\begin{tabular}{cccccccc}
\hline $\begin{array}{c}\text { Séries } \\
\text { em nível }\end{array}$ & \multicolumn{3}{c}{ Modelos ADF $^{(\mathrm{a})}$} & \multicolumn{5}{c}{$\begin{array}{c}\text { Séries em } \\
\text { primeira } \\
\end{array}$} & $\mathrm{I}$ & $\mathrm{II}^{(\mathrm{b})}$ & $\mathrm{III}$ & $\begin{array}{c}\text { Modelos ADF } \\
\text { diferença }\end{array}$ & $\mathrm{I}$ & $\mathrm{II}$ & $\mathrm{III}$ \\
\hline LPSP & $-1,6839$ & $-1,9020$ & 0,1269 & DLPSP & $-11,5919$ & $-11,5390$ & $-11,5787$ \\
LPCE & $-3,0271$ & $-3,4367$ & 0,0966 & DLPCE & $-9,7380$ & $-9,7255$ & $-9,6728$ \\
LPPI & $-1,7796$ & $-2,1234$ & 0,2735 & DLPPI & $-17,0347$ & $-16,9583$ & $-17,0154$ \\
LPSC & $-1,6825$ & $-1,8196$ & 0,4029 & DLPSC & $-15,8609$ & $-15,7937$ & $-15,7984$ \\
LPRS & $-1,5784$ & $-1,7454$ & 0,3324 & DLPRS & $-14,3099$ & $-14,2476$ & $-14,2778$ \\
\hline & & \multicolumn{7}{c}{ Phillips-Perron (PP) ${ }^{(\mathrm{c})}$} & & & \\
\hline LPSP & $-2,7938$ & $-3,4893$ & $-0,2444$ & DLPSP & $-17,6650$ & $-17,5752$ & $-17,6067$ \\
LPCE & $-2,7345$ & $-3,1508$ & $-0,1241$ & DLPCE & $-15,2266$ & $-15,0897$ & $-14,9000$ \\
LPPI & $-2,5026$ & $-3,1380$ & 0,2097 & DLPPI & $-18,0494$ & $-17,9631$ & $-17,7573$ \\
LPSC & $-1,9675$ & $-2,3513$ & $-0,2240$ & DLPSC & $-15,5999$ & $-15,5372$ & $-15,3731$ \\
LPRS & $-2,0164$ & $-2,2812$ & $-0,0976$ & DLPRS & $-14,2372$ & $-14,1761$ & $-14,0440$ \\
\hline
\end{tabular}

Fonte: Elaborado pelos autores com base nos resultados da pesquisa.

(a) Os valores críticos ao nível de significância de $1 \%$ são $-3,4896 ;-4,0420$; e $-2,5857$, respectivamente, nos modelos I, II e III. ${ }^{\text {(b) }} \mathrm{O}$ p-valor da tendência de LPSP é 0,3744 ; LPCE é 0,1081 ; LPPI é 0,2501 ; LPSC é 0,4597; e LPRS é $0,4444 .{ }^{(c)}$ Os valores críticos ao nível de significância de $1 \%$ são - 3,4891; -4,0412; e 2,5855, respectivamente, nos modelos I, II e III. 
Conforme se verifica pela Tabela 2, o teste KPSS também reitera que todas as séries são estacionárias em primeira diferença. Portanto, de acordo com os três testes de raízes unitárias, as séries de preços estudadas são integradas de ordem um.

Tabela 2 - Resultados do teste KPSS em primeira diferença para as séries mensais de preços logaritmizadas do mel natural brasileiro exportado pelos estados considerados na análise, janeiro de 2002 a julho de 2011.

\begin{tabular}{ccc}
\hline Séries & \multicolumn{3}{c}{ Modelos $^{(\text {a) }}$} & II \\
\hline DLPSP & 0,0817 & 0,0859 \\
DLPCE & 0,1072 & 0,0997 \\
DLPPI & 0,0957 & 0,0974 \\
DLPSC & 0,0766 & 0,0804 \\
DLPRS & 0,0863 & 0,0922 \\
\hline
\end{tabular}

Fonte: Elaborado pelos autores com base nos resultados da pesquisa.

(a) $\mathrm{O}$ modelo I corresponde ao modelo apenas com intercepto e seu valor crítico ao nível de significância de $1 \%$ é 0,7390 ; e o modelo II refere-se ao modelo com intercepto e tendência e seu valor crítico ao nível de significância de $1 \%$ é 0,2160 .

Com o intuito de obter resultados dos testes de raiz unitária mais robustos, empregou-se o teste HEGY de raiz unitária sazonal. Na especificação do teste, foi incluído um termo de intercepto e as defasagens foram escolhidas por meio do critério de Schwartz. Os dados da Tabela 3 evidenciam o indicativo de ausência de raízes unitárias sazonais em todas as séries, conforme mostrada pela significância estatística de $\pi$. Além disso, não se identificou raiz unitária semi-anual nem raízes unitárias complexas nos pares examinados de $\pi \mathrm{i}$ 's. Desta forma, os preços do mel natural exportado pelo Brasil nos estados analisados foram estacionários em primeira diferença, rejeitando a hipótese de raiz unitária sazonal.

Tabela 3 - Teste de identificação da presença de raízes sazonais nas séries de preços mensais do mel natural exportado pelos estados considerados na análise, janeiro de 2002 a julho de 2011.

\begin{tabular}{|c|c|c|c|c|c|}
\hline Frequência & & & Séries & & \\
\hline Coeficientes & LPSP & LPCE & LPPI & LPSC & LPRS \\
\hline Lags $^{(a)}$ & 0 & 0 & 0 & 0 & 0 \\
\hline $0 / \pi_{1}$ & $2,35^{(\mathrm{b})}$ & $2,61^{(\mathrm{b})}$ & $2,39^{(\mathrm{b})}$ & $2,80^{(\mathrm{b})}$ & $1,87^{(\mathrm{b})}$ \\
\hline$\pi / \pi_{2}$ & $-1,64^{*}$ & $-2,64 * * *$ & 1,44 & $-2,41 * *$ & $-2,48 * *$ \\
\hline$\frac{\pi}{2} / \pi_{3}$ e $\pi_{4}$ & 0,55 & $4,22 * *$ & 1,40 & $6,59 * * *$ & $6,00 * * *$ \\
\hline$\frac{2 \pi}{3} / \pi_{5} \mathrm{e} \pi_{6}$ & $10,68 * *$ & $12,58 * * *$ & $17,41 * * *$ & $17,84 * * *$ & $14,42 * * *$ \\
\hline$\frac{\pi}{3} / \pi_{7}$ e $\pi_{8}$ & $7,94 * * *$ & $12,92 * * *$ & $10,78 * * *$ & $11,06 * * *$ & $10,10 * * *$ \\
\hline$\frac{5 \pi}{6} / \pi_{9}$ e $\pi_{10}$ & $10,42 * * *$ & $13,87 * * *$ & $14,30 * * *$ & $12,47 * * *$ & $9,80 * * *$ \\
\hline
\end{tabular}




\begin{tabular}{llllll}
$\frac{\pi}{6} / \pi_{11}$ e $\pi_{12}$ & $10,13 * * *$ & $8,79 * * *$ & $10,57 * * *$ & $13,99 * * *$ & $9,68 * * *$ \\
$\begin{array}{c}\pi_{1}, \ldots, \pi_{12-} \\
\quad \text { Complexa }\end{array}$ & $8,13 * * *$ & $36,94 * * *$ & $30,42 * * *$ & $57,09 * * *$ & $62,65 * * *$ \\
$\begin{array}{c}\pi_{2}, \ldots, \pi_{12}- \\
\text { Complexa }\end{array}$ & $8,38 * * *$ & $40,29 * * *$ & $33,18 * * *$ & $62,26 * * *$ & $68,34 * * *$ \\
\hline
\end{tabular}

Fonte: Elaborado pelos autores com base nos resultados da pesquisa.

Nota: ${ }^{(a)} \mathrm{O}$ número de defasagens foi baseado no critério de Schwarz; ${ }^{(b)}$ Indica não rejeição da hipótese nula; (***), (**) e (*) indicam rejeição de $\mathrm{H}_{0}$ a $1 \%, 5 \%$ e $10 \%$, respectivamente, cujos valores críticos foram obtidos por Franses e Hobijn (1990).

\subsection{Vetor Auto-Regressivo (VAR)}

Como se verifica pelos testes de raiz unitária, as séries analisadas dos preços do mel natural exportado nos estados de São Paulo, Ceará, Piauí, Santa Catarina e Rio Grande do Sul são integradas na mesma ordem. Neste caso, pode-se passar para a análise de cointegração, em que se empregou o teste de Johansen, com o intuito de verificar se elas possuem relacionamento de longo prazo. Entretanto, para se testar a cointegração, deve-se inicialmente identificar o número de defasagens que deve ser incluído no modelo VAR. A escolha do número de defasagens foi baseada nos critérios Akaike (AIC), Schawarz (SC) e HannanQuinn (HQ).

Os resultados da Tabela 4 sinalizam que os dois últimos critérios (SC e HQ) apontaram para uma defasagem em todas as séries analisadas, com exceção da relação de preços entre os estados de Santa Catarina e Rio Grande do Sul, sendo, portanto, escolhida uma defasagem no modelo VAR. No caso de LPSC x LPRS, observa-se um impasse na escolha da determinação do número de defasagens, pois cada critério indica um número diferente de lags. Neste caso, seguiu-se o critério de Schawarz, que é o mais parcimonioso, e também se opta por uma defasagem.

Tabela 4 - Determinação do número de defasagens para as séries de preços mensais do mel natural exportado pelos estados considerados na análise, janeiro de 2002 a julho de 2011.

\begin{tabular}{ccccc}
\hline Séries relacionadas & Lag & AIC & SC & HQ \\
\hline LPSP x LPRS & 1 & $-2,8598$ & $-2,7099^{*}$ & $-2,7990^{*}$ \\
& 2 & $-2,8803^{*}$ & $-2,6305$ & $-2,7791$ \\
\hline LPCE x LPRS & 1 & $-3,4630$ & $-3,3131^{*}$ & $-3,4022^{*}$ \\
& 2 & $-3,4875^{*}$ & $-3,2377$ & $-3,3862$ \\
\hline LPPI x LPRS & 1 & $-3,6317$ & $-3,4818^{*}$ & $-3,5710^{*}$ \\
& 2 & $-3,6603^{*}$ & $-3,4106$ & $-3,5591$ \\
\hline \multirow{2}{*}{ LPSC x LPRS } & 1 & $-4,1335$ & $-3,9836^{*}$ & $-4,0728$ \\
& 2 & $-4,1850$ & $-3,9352$ & $-4,0837^{*}$ \\
& 4 & $-4,1964^{*}$ & $-3,7468$ & $-4,0141$ \\
\hline
\end{tabular}

Fonte: Elaborado pelos autores com base nos resultados da pesquisa.

* Indica a ordem selecionada pelo critério. 


\subsection{Vetor de Correção de Erros (VEC) e testes de hipóteses sobre os parâmetros $\beta$ e $\alpha$}

Como os valores das probabilidades do componente de tendência não foram significativos nas séries de preço analisadas, considerou-se a especificação que não apresenta tendência determinística. Ademais, adotou-se que a constante está incorporada dentro do termo de correção de erro.

Os resultados dos testes do traço e do máximo autovalor mostrados na Tabela 5 indicam que a hipótese nula de que não há nenhum vetor de cointegração foi rejeitada em nível de 5\% nos pares de séries analisados, logo existe um vetor de cointegração em cada par de séries de preços do mel natural exportado. Esse resultado da presença de pelo menos um vetor de cointegração assegura a existência de um vetor de correção de erros. Desta forma, foram estimados os coeficientes de ajuste de curto e de longo prazo (Tabelas 6 e 7).

Tabela 5 - Resultados do teste de cointegração de Johansen para as séries de preços mensais do mel natural exportado pelos estados considerados na análise, janeiro de 2002 a julho de 2011.

\begin{tabular}{cccccc}
\hline Séries relacionadas & $\begin{array}{c}\text { Hipótese } \\
\text { nula }\end{array}$ & $\begin{array}{c}\text { Teste do } \\
\text { Traço }\end{array}$ & $\begin{array}{c}\text { Valor } \\
\text { Crítico } \\
(5 \%)\end{array}$ & $\begin{array}{c}\text { Teste do máximo } \\
\text { autovalor }\end{array}$ & $\begin{array}{c}\text { Valor Crítico } \\
(5 \%)\end{array}$ \\
\hline LPSP x LPRS & $\mathrm{r}=0$ & $68,2960^{*}$ & 12,3209 & $68,2941^{*}$ & 11,2248 \\
& $\mathrm{r} \leq 1$ & 0,0019 & 4,1299 & 0,0019 & 4,1299 \\
\hline LPCE x LPRS & $\mathrm{r}=0$ & $50,2131^{*}$ & 12,3209 & $50,2101^{*}$ & 11,2248 \\
& $\mathrm{r} \leq 1$ & 0,0029 & 4,1299 & 0,0029 & 4,1299 \\
\hline LPPI x LPRS & $\mathrm{r}=0$ & $67,9233^{*}$ & 12,3209 & $67,9198^{*}$ & 11,2248 \\
& $\mathrm{r} \leq 1$ & 0,0035 & 4,1299 & 0,0035 & 4,1299 \\
\hline LPSC x LPRS & $\mathrm{r}=0$ & $59,8895^{*}$ & 12,3209 & $59,8868^{*}$ & 11,2248 \\
& $\mathrm{r} \leq 1$ & 0,0027 & 4,1299 & 0,0027 & 4,1299 \\
\hline
\end{tabular}

Fonte: Elaborado pelos autores com base nos resultados da pesquisa.

* Indica rejeição da hipótese nula a 5\% de significância.

As equações de equilíbrio de longo prazo para cada par de séries de preços do mel natural exportado estão apresentadas na Tabela 6. Tais equações indicam que, mantendo tudo mais constante, cada variação de $1 \%$ no preço do mel natural exportado pelo Rio Grande do Sul ocasiona, por exemplo, uma variação de $0,976 \%$ no preço do mel natural exportado pelo Piauí e uma variação de $0,996 \%$ no preço da soja no preço do mel natural exportado por Santa Catarina, no período de janeiro de 2002 a julho de 2011. Isso significa que 97,64\% e 99,59\% das variações de preços, no longo prazo, ocorridas no Rio Grande do Sul, são transmitidas para o preço do mel natural exportado pelo Piauí e Santa Catarina, respectivamente. Essa interpretação pode ser replicada para as demais equações. 
Tabela 6 - Equações de equilíbrio de longo prazo para as séries de preços mensais do mel natural exportado pelos estados considerados na análise, janeiro de 2002 a julho de 2011.

$\begin{array}{cc}\text { Séries relacionadas } & \text { Equação de equilíbrio de longo } \\ \text { LPSP x LPRS } & \text { LPSP }=1,0325 \mathrm{LPRS} \\ \text { LPCE x LPRS } & \text { LPCE }=1,0820 \mathrm{LPRS} \\ \text { LPPI x LPRS } & \text { LPPI }=0,9764 \mathrm{LPRS} \\ \text { LPSC } x \text { LPRS } & \text { LPSC }=0,9959 \mathrm{LPRS}\end{array}$

Fonte: Elaborado pelos autores com base nos resultados da pesquisa.

Segundo Freitas et al. (2001), se duas variáveis são cointegradas, elas convergem para uma condição de equilíbrio de longo prazo, tornando-se importante estimar o Modelo de Correção de Erro (VEC), já que este modelo possibilita determinar a velocidade com a qual as variáveis tendem a obter o equilíbrio no longo prazo, ao incluir componentes de curto prazo e de longo prazo, sendo que tais aspectos de longo prazo são captados por meio dos resíduos defasados da equação de cointegração.

Conforme se verifica pela Tabela 7, os parâmetros de correção do desequilíbrio de curto prazo referente à trajetória de longo prazo referente ao Rio Grande do Sul quando se considera os pares de séries entre São Paulo e Rio Grande do Sul e entre Ceará e Rio Grande do Sul mostraram-se estatisticamente significantes ao nível de 10\%. Isso indica que variações de curto prazo, nas trajetórias de equilíbrio do preço do mel natural exportado entre São Paulo e Rio Grande do Sul são corrigidas no próximo mês em 12,36\%, ou seja, precisaria, em média, de oito meses para remover os desequilíbrios, ao passo que desvios das trajetórias entre Ceará e Rio Grande do Sul são corrigidos no próximo mês em 11,25\%, necessitando, em média, de quase nove meses para corrigir o desequilíbrio.

Tabela 7 - Estimação do VEC referente à variável preço do mel natural em São Paulo, Ceará, Piauí, Santa Catarina e Rio Grande do Sul, janeiro de 2002 a julho de 2011.

\begin{tabular}{ccc}
\hline LPSP x LPRS & $\Delta$ LPSP $t$ & $\Delta$ LPRS $t$ \\
\hline Variável $\alpha$ & $-0,7708$ & 0,1236 \\
& {$[-7,4024](0,1041)$} & {$[1,7225](0,0718)$} \\
\hline LPCE x LPRS & $\Delta$ LPCE $t$ & $\Delta \mathrm{LPRS} t$ \\
\hline Variável $\alpha$ & $-0,5858$ & 0,1125 \\
& {$[-5,2574](0,1114)$} & {$[1,3252](0,0849)$} \\
\hline LPPI x LPRS & $\Delta \mathrm{LPPI} t$ & $\Delta \mathrm{LPRS} t$ \\
\hline Variável $\alpha$ & $-0,8061$ & 0,0995 \\
& {$[-6,2612](0,1287)$} & {$[0,9250](0,1075)$} \\
\hline LPSC x LPRS & $\Delta \mathrm{LPSC} t$ & $\Delta \mathrm{LPRS} t$ \\
\hline Variável $\alpha$ & $-0,6933$ & 0,1042 \\
& {$[-5,1167](0,1355)$} & {$[0,7939](0,1313)$} \\
\hline
\end{tabular}

Fonte: Elaborado pelos autores com base nos resultados da pesquisa. 
Nota: Os valores entre colchetes referem-se à estatística t e os valores entre parênteses correspondem ao erro padrão.

Tendo em vista que as elasticidades de transmissão de preços mostradas na Tabela 6 foram próximas da unidade, então a Lei do Preço Único predomina nesses mercados. Entretanto, segundo Costa e Ferreira Filho (2000), Barbosa et al. (2002) e Coelho (2004), é necessário impor restrições aos parâmetros $\beta$ para verificar se essa lei permanecerá. A Tabela 8 apresenta os resultados dos testes de hipóteses sobre os parâmetros $\beta$ com o intuito de identificar as variáveis que participam efetivamente do equilíbrio de longo prazo e verificar o grau de integração entre os pares de mercados analisados. Com base nesses resultados, todos os preços efetivamente participavam do equilíbrio de longo prazo.

Como os mercados brasileiros exportadores de mel natural são integrados, é relevante testar a hipótese de perfeita integração. As informações da Tabela 8 indicam que não há perfeita integração entre os mercados considerados. Desta forma, rejeitou-se a hipótese nula de perfeita integração dos mercados para todas as relações consideradas. Com isso, pode-se inferir que alterações de preços de exportação do mel natural em um mercado não são completamente transmitidas aos demais mercados no longo prazo, sinalizando que a LPU não é perfeitamente verificada para os mercados de mel natural no período analisado.

Tabela 8 - Teste de significância de restrição ao parâmetro de longo prazo $(\beta)$ dos vetores de cointegração das séries de preços mensais do mel natural exportado pelos estados considerados na análise, janeiro de 2002 a julho de 2011.

\begin{tabular}{cccc}
\hline Séries relacionadas & Hipótese nula & Razão de Verossimilhança & Valor crítico (5\%) \\
\hline \multirow{2}{*}{ LPSP x LPRS } & $\beta_{\mathrm{SP}}=0$ & $68,2381^{*}$ & 3,84 \\
& $\beta_{\mathrm{RS}}=0$ & $67,2983^{*}$ & 3,84 \\
& $\beta_{\mathrm{SP}}=\beta_{\mathrm{RS}}$ & $68,1359^{*}$ & 3,84 \\
\hline \multirow{2}{*}{ LPCE x LPRS } & $\beta_{\mathrm{CE}}=1$ & $50,1594^{*}$ & 3,84 \\
& $\beta_{\mathrm{RS}}=1$ & $49,7585^{*}$ & 3,84 \\
& $\beta_{\mathrm{CE}}=\beta_{\mathrm{RS}}$ & $50,1474^{*}$ & 3,84 \\
\hline \multirow{2}{*}{ LPPI x LPRS } & $\beta_{\mathrm{PI}}=1$ & $67,8237^{*}$ & 3,84 \\
& $\beta_{\mathrm{RS}}=1$ & $67,5869^{*}$ & 3,84 \\
& $\beta_{\mathrm{PI}}=\beta_{\mathrm{RS}}$ & $67,8993^{*}$ & 3,84 \\
\hline \multirow{2}{*}{ LPSC x LPRS } & $\beta_{\mathrm{SC}}=1$ & $59,7249^{*}$ & 3,84 \\
& $\beta_{\mathrm{RS}}=1$ & $59,8083^{*}$ & 3,84 \\
& $\beta_{\mathrm{SC}}=\beta_{\mathrm{RS}}$ & $59,8801^{*}$ & 3,84 \\
\hline
\end{tabular}

Fonte: Elaborado pelos autores com base nos resultados da pesquisa.

* Indica rejeição ao nível de 5\% de significância.

Ademais, também se testou a hipótese sobre o parâmetro $\alpha$ (exogeneidade fraca) para verificar qual mercado, dentre os analisados, pode ser considerado como exógeno fraco, assim 
como testar as velocidades de resposta das variáveis a uma dada situação de desequilíbrio de curto prazo no processo de ajuste de longo prazo. Os resultados mostrados na Tabela 9 revelaram a não rejeição da hipótese nula para o preço do mel natural no estado do Rio Grande do Sul, sinalizando como incapaz de ser influenciado no longo prazo pelos demais mercados enfocados. Desta forma, o estado gaúcho pode ser considerado exógeno fraco.

Observou-se ainda configuração diferenciada para as regiões paulista, cearense, piauiense e catarinense, em que foi rejeitada a hipótese nula de mercado exógeno fraco, com probabilidade a $10 \% ; 5 \% ; 1 \%$; e $1 \%$, respectivamente. Portanto, constata-se que o estado do Rio Grande do Sul atua como mercado central, capaz de influenciar mudanças nos preços do mel natural dos estados de São Paulo, Ceará, Piauí e Santa Catarina, no longo prazo. Esse resultado pode ser atribuído ao fato do estado gaúcho ter representado a maior variação percentual de crescimento do total de mel natural exportado no período enfocado; e historicamente por esse Estado ter se destacado como líder brasileiro da produção de mel.

Tabela 9 - Teste de exogeneidade fraca realizado para as variáveis LPSP, LPCE, LPPI, LPSC e LPRS, janeiro de 2002 a julho de 2011.

\begin{tabular}{ccc}
\hline Mercados potencialmente & Estatística de teste & P-valor \\
exógenos & $3,5455^{*}$ & 0,0597 \\
São Paulo (LPSP) & $4,8961^{* *}$ & 0,0269 \\
Ceará (LPCE) & $22,4341^{* * *}$ & 0,0000 \\
Piauí (LPPI) & $11,8337^{* * *}$ & 0,0006 \\
Santa Catarina (LPSC) & $0,0831^{\mathrm{NR}}$ & 0,7732 \\
Rio Grande do Sul (LPRS) & & .
\end{tabular}
Notas: ${ }^{\mathrm{NR}}$ Indica não rejeição da hipótese nula a $10 \% ;(* * *),(* *)$ e $(*)$ indicam rejeição de $\mathrm{H}_{0}$ a $1 \%$, $5 \%$ e $10 \%$, respectivamente. Fonte: Elaborado pelos autores com base nos resultados da pesquisa.

No tocante à hipótese que analisa as velocidades de resposta das variáveis a uma dada situação de desequilíbrio de curto prazo no processo de ajuste de longo prazo, os dados da Tabela 10 revelam que elas não são estatisticamente iguais para os pares de mercados analisados.

Tabela 10 - Testes de significância de restrição sobre o parâmetro $\alpha$ para os pares de mercados considerados.

\begin{tabular}{ccc}
\hline Hipótese nula & Razão de Verossimilhança & Valor Crítico (5\%) \\
\hline$\alpha L P S P=\alpha L P R S$ & $68,1959^{*}$ & 3,84 \\
$\alpha L P C E=\alpha L P R S$ & $50,2060^{*}$ & 3,84 \\
$\alpha L P P I=\alpha L P R S$ & $67,4046^{*}$ & 3,84 \\
$\alpha L P S C=\alpha L P R S$ & $57,6189^{*}$ & 3,84 \\
\hline
\end{tabular}

Nota: * Indica rejeição a 5\% de significância

Fonte: Elaborado pelos autores com base nos resultados da pesquisa. 


\section{CONSIDERAÇÕES FINAIS}

Os resultados indicam a presença de relações de equilíbrio de longo prazo entre os preços de exportação do mel natural praticados entre os estados de São Paulo, Ceará, Piauí e Santa Catarina com o Rio Grande do Sul.

As elasticidades de transmissão de preços entre os mercados de mel natural analisados indicam que variações em um mercado, em longo prazo, foram repassadas quase que totalmente para o outro mercado analisado durante o período de janeiro de 2002 a julho de 2011, sinalizando a predominância da Lei do Preço Único nesses mercados. Entretanto, tais mercados não podem ser considerados perfeitamente integrados, já que a hipótese de perfeita integração entre eles foi rejeitada. Portanto, a LPU não é perfeitamente verificada para o mercado exportador de mel natural nos estados analisados e no período considerado.

Com base no teste de exogeneidade fraca, verifica-se que o estado do Rio Grande do Sul atua como mercado central, podendo ser atribuído ao fato de esse Estado ter representado a maior variação percentual de crescimento do total de mel natural exportado no período enfocado. Ademais, esse Estado tem se destacado historicamente como líder brasileiro da produção de mel.

Finalmente, é importante destacar que este trabalho analisou o processo de integração espacial entre os principais mercados brasileiros exportadores de mel natural sem considerar a presença de custos de transação, porém, em estudos posteriores, sugere-se que tais custos sejam incorporados, assim como que se verifique a transmissão de preços do mel natural entre os principais países exportadores e o mercado brasileiro.

\section{REFERÊNCIAS}

AMORIM, A. L.; SOUSA, E. P.; CORONEL, D. A. Preço da cesta básica na Região Sul do Brasil: testando a integração espacial. Revista de Economia e Administração, São Paulo, v. 12, n. 3, p. 321-334, 2013.

ARDENI, P. G. Does the Law of One Price really hold for commodity prices? American Journal of Agricultural Economics, Oxford, v. 71, n. 3, p. 661-669, 1989.

BARBOSA, M. Z.; MARGARIDO, M. A.; NOGUEIRA JÚNIOR, S. Análise da elasticidade de transmissão de preços no mercado brasileiro de algodão. Nova Economia, Belo Horizonte, v. 12, n. 2, p. 79-108, 2002. 
CHIODI, L. Integração espacial no mercado brasileiro de milho. 2006.89 f. Dissertação (Mestrado em Economia) - Programa de Pós-Graduação em Economia Aplicada, Universidade de São Paulo, Piracicaba, SP, 2006.

COELHO, A. B. A cultura do algodão e a questão da integração entre preços internos e externos. Revista de Economia e Sociologia Rural, Rio de Janeiro, v. 42, n. 1, p. 153-169, 2004.

CORONEL, D. A. et al. Integração e transmissão de preços entre os mercados de trigo argentino e internacional. Revista Pesquisa \& Debate, São Paulo, v. 21, n. 2, p. 279-305, 2010 .

COSTA, S. M. A. L.; FERREIRA FILHO, J. B. S. Liberalização comercial no Brasil e integração nos mercados de commodities agrícolas: os mercados de algodão, milho e arroz. Revista de Economia e Sociologia Rural, Rio de Janeiro, v. 38, n. 2, p. 41-70, 2000.

CUNHA, D. A. Integração de preços no mercado internacional de café. 2008. $123 \mathrm{f}$. Dissertação (Mestrado em Economia Aplicada) - Universidade Federal de Viçosa, Viçosa, MG, 2008.

DICKEY, D. A.; FULLER, W. Likelihood ratio statistics for autoregressive time series with a unit root. Econometrica, New York, v. 49, n. 4, p. 1057-1072, 1981.

ENDERS, W. Applied econometric time series. New York: John Wiley and Sons, 1995.

FAMINOW, M. D.; BENSON, B. L. Spatial market integration. American Journal of Agricultural Economics, Oxford, v. 72, n. 1, p. 49-62, 1990.

FACKLER, P. L.; GOODWIN, B. K. Spatial price analysis. IN: RAUSSER, G. C.; GARDEN, B. L. (Eds.). Handbook of Agricultural Economics. Amsterdam: North-Holland Press, 2001. p. 971-1024.

FAMINOW, M. D.; BENSON, B. L. Spatial market integration. American Journal of Agricultural Economics, Oxford, v. 72, n. 1, p. 49-62, 1990.

FAO - Food Agriculture Organization United Nations. FAOstat. Disponível em: <http://faostat.fao.org/default.aspx>. Acesso em: 18 fev. 2011.

FRANSES, P. H.; HOBIJN, B. Critical values for unit root tests in seasonal time series. Journal of Applied Statistics, Abingdon, v. 24, p. 25-46, 1997.

FREITAS, C. O.; LÍRIO, V. S.; CORONEL, D. A. Análise da transmissão de preço do fumo entre os estados produtores brasileiros e no mercado internacional. Revista Ciências Administrativas, Fortaleza, v. 18, n. 1, p. 13-44, 2012.

FREITAS, D. G. F.; KHAN, A. S.; SILVA, L. M. R. Nível tecnológico e rentabilidade de produção de mel de abelha (Apis Mellifera) no Ceará. Revista de Economia e Sociologia Rural, Rio de Janeiro, v. 42, n. 1, p. 171-188, 2004. 
FREITAS, S. M. et al. Análise da dinâmica de transmissão de preços no mercado internacional de farelo de soja, 1990-99. Agricultura em São Paulo, São Paulo, v. 48, n. 1, p. $1-20,2001$.

HAUER, M. Os modelos VAR e VEC espaciais: uma abordagem bayesiana. $201 \mathrm{f}$. Dissertação (Mestrado em Economia) - Universidade Federal do Rio Grande do Sul, Porto Alegre, 2007.

HYLLEBERG. S. et al. Seasonal integration and cointegration. Journal of Econometrics, v. 44, n. 1-2, p. 215-238, 1990.

JOHANSEN, S. JUSELIUS, K. Maximum likelihood estimation and inference on cointegration with applications to the demand for Money. Oxford Bulletin of Economics and Statistics, Hoboken, v. 52, n. 2, p. 169-210, 1990.

KRUGMAN, P. R.; OBSTFELD, M. International economics: theory and policy. 5. ed. Massachusetts: Addson Welsley, 2005.

MARGARIDO, M. A; BARROS, G. S. C. Transmissão de preços agrícolas internacionais para preços agrícolas domésticos no Brasil. Agricultura, São Paulo, v. 2, n. 47, p. 53-81, 2000 .

MARGARIDO, M. A.; MEDEIROS JUNIOR, H. Teste para mais de uma raiz unitária: uso do software $\mathrm{SAS} \circledast$ na elaboração de uma rotina para o teste Dickey Pantula. Pesquisa \& Debate, São Paulo, v. 17, n. 1, p. 149-170, 2006.

MAYORGA, R. O. Análise de transmissão de preços do mercado atacadista de melão do Brasil. 2006. 94 f. Dissertação (Mestrado em Economia Rural) - Departamento de Economia Agrícola, Universidade Federal do Ceará, Fortaleza, CE, 2006.

MATOS, V. D. A apicultura no estado do Ceará: competitividade, nível tecnológico e seus fatores condicionantes, produção e exportação de mel natural. 2005. 192 f. Dissertação (Mestrado em Economia Rural) - Universidade Federal do Ceará, Fortaleza, CE, 2005.

MDIC - Ministério de Desenvolvimento Indústria e Comércio / SECEX - Secretaria de Comércio Exterior. Valores e quantidades anuais de mel natural exportados no mercado: janeiro/1986 a dezembro/2010. Brasília, DF. Disponível em:

<aliceweb.desenvolvimento.gov.br>. Acesso em: 10 set. 2011.

MEYER, J. Measuring market integration in the presence of transaction costs - a threshold vector error correction approach. Agricultural Economics, Hoboken, v. 31, p. 327-334, 2004.

MÓL, A. L. R.; ALDATZ, R. J. Integração espacial no mercado de camarão. Custos e @ gronegócio on line, Recife, v. 10, n. 1, p. 29-54, 2014. 
MORAES, M. L. Integração espacial no mercado brasileiro de etanol. 2014. 130 f. Tese (Doutorado em Ciências com área de concentração em Economia Aplicada) - Universidade de São Paulo, Escola Superior de Agricultura “Luiz de Queiróz", Piracicaba, SP, 2014.

NOGUEIRA, F. T. P.; AGUIAR, D. R. D.; LIMA, J. E. Integração espacial no mercado brasileiro de café arábica. Nova Economia, Belo Horizonte, v. 15, n. 2, p. 91-112, 2005.

ROSADO, P. L. Integração espacial entre os mercados brasileiros de suínos. 2006. 135 f. Tese (Doutorado em Economia Rural) - Universidade Federal de Viçosa, Viçosa, MG, 2006.

SOUSA, E. P.; CAMPOS, A. C. Transmissão de preços do algodão nos mercados interno e externo. Revista de Política Agrícola, Brasília, DF, v. 17, n. 3, p. 5-16, 2008.

SOUSA, E. P.; CAMPOS, A. C. Transmissão inter-regional de preços no mercado brasileiro de soja. Revista Análise Econômica, Porto Alegre, v. 27, n. 51, p. 83-105, 2009. 